\title{
The Effect of Intracranial Migration of Foley Balloon Catheter after Tumor Resection
}

\author{
Lijun Yang, $M D, P h D^{1}$, Tongmao $W u, M D^{1}$, Junling Cui, $M D^{1}$, Feng Wang, $M D^{1}$, Jianliang $W u, M D, P h D^{1}$, \\ Gengshen Zhang, $M D, P h D^{1}$ and Zhenzeng Fan, $M D, P h D^{1^{*}}$
}

Department of Neurosurgery, The Second Hospital of Hebei Medical University, China

\begin{abstract}
Object: The incidence of intracranial tumor is $5 \%$ of that of systemic tumor. Some of them are presented as large ones because of slow growth. The higher complications are challenging due to their potential behaviors after resection such as large residual cavity, cortex collapse and bridging vein rupture.

Methods: Totally 181 cases of large intracranial tumors that undergone the migration of Foley balloon catheter between Jan 2011 and Jan 2014 were enrolled in this study. The sizes of tumor, the volume of saline injection, extubation time and location were all reviewed retrospectively.

Results: Foley balloon catheters were implanted in all the patients after the tumor resection. There were 112 women and 69 men with the mean age of $53.51 \pm 9.45$ years (range, 30-71 years). 129 presented with meningioma and 52 with glioma, 85 (65.89\%) meningiomas were found at the supratentorial and $44(34.11 \%)$ at subtentorial, $46(88.46 \%)$ gliomas were found at the supratentorial and $6(11.54 \%$ ) at subtentorial. The mean values of size were $40.71 \pm 20.45$ (range, $45-59 \mathrm{~mm}$ ) and 38.8 \pm 16.78 (range, $45-59 \mathrm{~mm}$ ) in meningioma and glioma respectively. The capsule volumes were $32.09 \pm 16.51 \mathrm{ml}$ vs. $29.71 \pm$ $13.81 \mathrm{ml}$ in meningioma and glioma. The volume of the capsule was smaller than that of the tumor itself respectively. The extubation time of meningioma was longer than that of glioma $(40.00 \pm 11.36 \mathrm{~h}$ vs. $16.5 \pm 6.38 \mathrm{~h}, \mathrm{p}<0.01)$.
\end{abstract}

Conclusion: Intracranial migration of Foley balloon catheter can effectively reduce the risk of postoperative complications such as intracranial hemorrhage, acute intracranial edema and ischemic cerebral infarction. The success of this technique in a series of our cases has encouraged its further use.

Keywords

Hematoma, Foley balloon catheter, Bridging vein, Giant intracranial tumor, CSF drainage

Abbreviations

SDH: Subdural Hematoma; EDH: Remote Epidural Hematoma; RCH: Residual Cavity Hematoma; CT: Computer Tomography; MRI: Magnetic Resonance Imaging; ICP: Intracranial Pressure; CSF: Cerebrospinal Fluid

\section{Introduction}

The total resection rate of large intracranial tumors was significantly improved with the great advances of microneurosurgical techniques and the application of a series of haemostatic materials [1]. Mortality and disability were significantly lower, however, postoperative complications, which are the main problems of neurosurgeon, are life-threatening events that clinicians need to take as a possible risk [2]. After the resection of giant intracranial tumor, there are many complications caused by residual cavity which include acute subdural hematoma (SDH), remote epidural hematoma (EDH), residual cavity hematoma ( $\mathrm{RCH})$, ischemic cerebral infarction, subcutaneous and tumor cavity tension effusion. A possible mechanism is proposed to explain this phenomenon and cavity-related complications have been successfully avoided by applying Foley balloon catheter after the resection of giant intracranial tumor.

\section{Materials and Methods}

\section{Patients and intracranial tumors}

This study was approved by the ethics committee on human research in the Second Hospital of Hebei Medical University. Informed consent was obtained from the patients or

*Corresponding author: Zhenzeng Fan, MD, PhD, Department of Neurosurgery, The Second Hospital of Hebei Medical University, No.36 Heping Road, Shijiazhuang City, Hebei Province, 050011, China, Tel: +86-18803110120

Accepted: July 11, 2019

Published online: July 13, 2019

Citation: Yang L, Wu T, Cui J, et al. (2019) The Effect of Intracranial Migration of Foley Balloon Catheter after Tumor Resection. J Neurosurg Res Rev 2(1):27-32 
their caregivers. 848 patients with intracranial tumors who entered into a prospectively controlled study in Hebei Medical University between Jan 2011 and Jan 2014 were retrospectively reviewed. The entry criteria of this study were as follows:

1) All intracranial tumors (>5.0 mm) including meningioma and glioma were confirmed by pathology postoperatively. The volume of the tumor was calculated using the following formula: $0.5 \times a \times b \times c$, where $a$ and $b$ are the largest diameters measured on Computer Tomography (CT) or Magnetic Resonance Imaging (MRI) and $\mathrm{c}$ is the slice thickness $(\mathrm{cm})$;

2) All the patients were reviewed by neurosurgery specialists at least twice to ensure that the entered data and diagnoses were complete and accurate;

3) Patients did not have the history of diabetes, hyperten- sion and have not undergone anticoagulant and anti-platelet treatment. Finally, 181 patients were found to meet the above conditions and their medical records were reviewed and presented in Table 1.

\section{Management and procedure}

All the patients received surgical operation with an appropriate surgical approach after general anesthesia. Following the completion of intracranial tumor resection, firstly, face the interior wall of residual cavity with oxidized cellulose Surgicel $^{\circledR}$ (Ethicon Inc., USA) after it is made sure that there is no "rolling-up smoke" by injecting saline into the cavity; secondly, cut the tip part of the Foley balloon catheter (22 Fr/ Ch, 50 CC, Kelong Medical, China) carefully without impairing the liquid capsule (Figure 1A); thirdly, test the safety of water capsule to ensure that there are no creases and it is

Table 1: Demography.

\begin{tabular}{|c|c|c|c|}
\hline & Meningioma $(n=129)$ & Glioma $(n=52)$ & P value \\
\hline Age, years & $53.76 \pm 9.45$ & $52.92 \pm 7.51$ & 0.324 \\
\hline \multicolumn{4}{|l|}{ Gender } \\
\hline M & $47 / 129$ & $22 / 52$ & \\
\hline $\mathbf{F}$ & $82 / 129$ & $30 / 52$ & 0.542 \\
\hline \multicolumn{4}{|l|}{ Location } \\
\hline Supratentorial & 85 (65.89\%) & 46 (88.46\%) & \\
\hline Subratentorial & 44 (34.11\%) & $6(11.54 \%)$ & $P<0.05$ \\
\hline Tumor volume (ml) & $40.71 \pm 20.45$ & $38.8 \pm 16.78$ & 0.355 \\
\hline Capsule volume (ml) & $32.09 \pm 16.51$ & $29.71 \pm 13.81$ & 0.843 \\
\hline Extubation time $(\mathrm{Hr})$ & $40.00 \pm 11.36$ & $16.5 \pm 6.38$ & $P<0.01$ \\
\hline
\end{tabular}

Indicates a statistically significant difference between groups $(P<0.05)$; $\mathrm{Hr}$ : Hour.
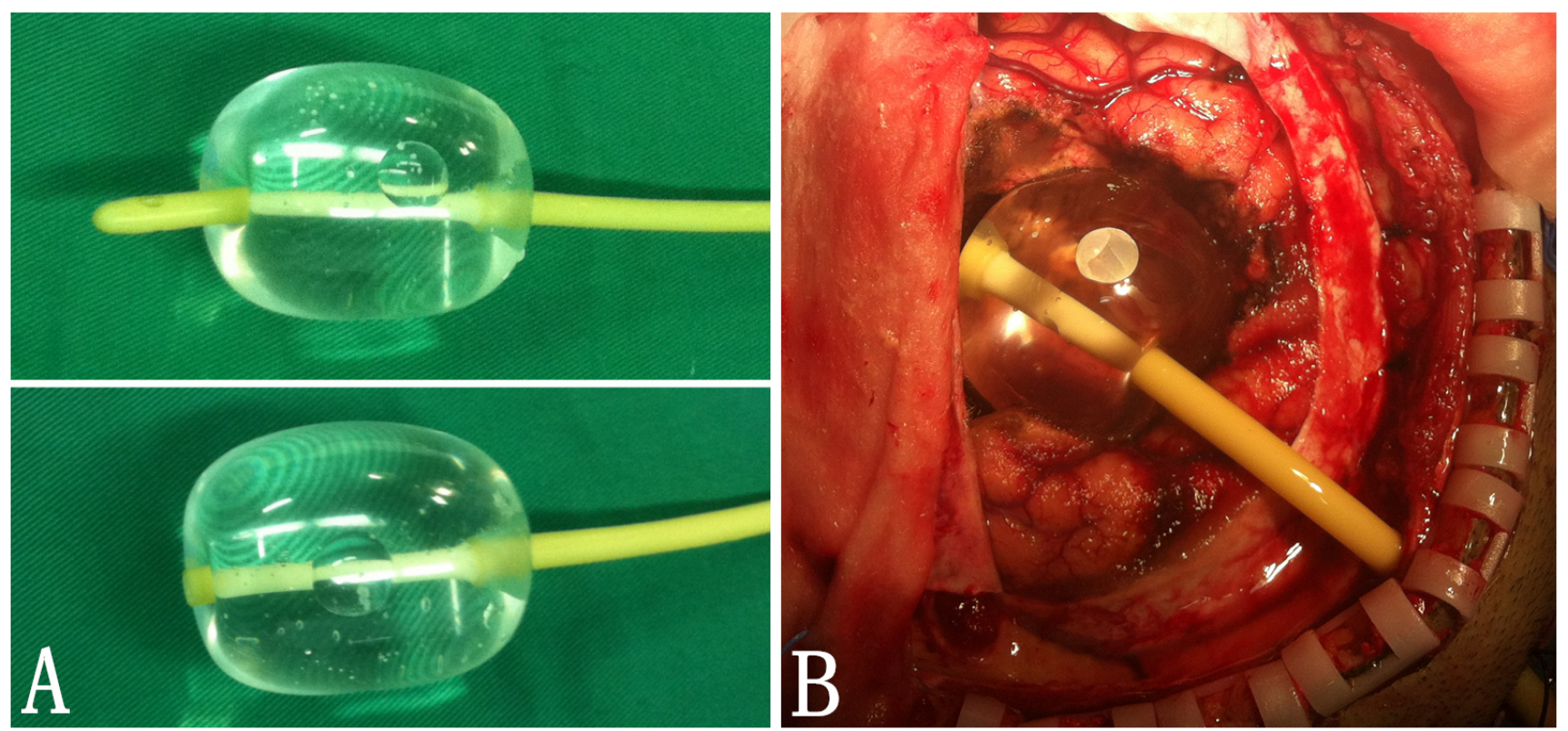

Figure 1: Intracranial migration of Foley balloon catheter during surgery after tumor resection.

A) (up) Foley balloon catheter was injected with $40 \mathrm{ml}$ saline, (down) the tip of the catheter was cut; B) A 14-F balloon catheter was filled with saline after positioned in the residual cavity. 
Citation: Yang L, Wu T, Cui J, et al. (2019) The Effect of Intracranial Migration of Foley Balloon Catheter after Tumor Resection. J Neurosurg Res $\operatorname{Rev} 2(1): 27-32$

Table 2: Summary of the surgical steps for intracranial migration of Foley balloon catheter.

\begin{tabular}{|l|l|}
\hline \multicolumn{2}{|l|}{ Surgical } \\
\hline 1. & Facing the interior wall of cavity of tumor with Surgicel ${ }^{\circledR}$. \\
\hline 2. & Cutting the tip part of the Foley balloon catheter carefully without impairing the liquid capsule. \\
\hline 3. & $\begin{array}{l}\text { Testing the safety of water capsule without creases to ensure that it is excessively transparent by priming with } 50 \mathrm{ml} \text { water } \\
\text { gradually and then swabbing off all the saline. }\end{array}$ \\
\hline 4. & Putting Foley balloon catheter into residual cavity then fill with $15-50 \mathrm{ml}$ saline according to the size of the residual cavity. \\
\hline
\end{tabular}

excessively transparent by priming with $50 \mathrm{ml}$ water gradually and then swabbing off; fourthly, make a subcutaneous tunnel to load drainage tube before Foley balloon catheter is fit into the residual cavity and filled with $15-50 \mathrm{ml}$ saline according to the size of the residual cavity (Figure 1B). The steps for intracranial migration of Foley balloon catheter was summarized in Table 2. Take record of the volume of saline that is injected into the cavity. Suture dura mater and close the skull under routine procedure. Take skull CT scan to view the postoperative situation 24 hours later. Record the time when the capsule is empty after around $10 \mathrm{ml}$ water is withdrawn every day.

\section{Statistical analyses}

Statistical analyses were performed using SPSS 15.0 statistical software (SPSS Inc, Chicago, IL). Continuous data were presented as mean \pm Standard deviation and compared between groups using the two-sample t-test. Categorical data were presented as numbers (\%) and were compared between groups using the Chi-square or Fisher's exact test. Statistical assessments were considered significant when $p<0.05$.

\section{Result}

All the patients were implanted with Foley balloon catheter after the tumor resection. There were 112 women and 69 men, with a mean age of $53.51 \pm 9.45$ years (range, 30-71 years). 129 presented with meningioma and 52 with glioma, $85(65.89 \%)$ meningiomas were found at the supratentorial and $44(34.11 \%)$ at subtentorial, $46(88.46 \%)$ gliomas were found at the supratentorial and $6(11.54 \%)$ at subtentorial. The mean size values were $40.71 \pm 20.45 \mathrm{~mm}$ and $38.8 \pm$ $16.78 \mathrm{~mm}$ in meningioma and glioma respectively. The capsule volumes were $32.09 \pm 16.51 \mathrm{ml}$ vs. $29.71 \pm 13.81 \mathrm{ml}$ in meningioma and glioma. The volume of the capsule is smaller than that of the tumor itself. The extubation time of meningioma is longer than that of glioma $(40.00 \pm 11.36 \mathrm{~h}$ vs. 16.5 $\pm 6.38 \mathrm{~h}, \mathrm{p}<0.01)$.

\section{Discussion}

The total resection rate of large intracranial tumors was significantly improved with the great advances of microneurosurgical techniques and the application of a series of haemostatic materials [1,3-5]. Mortality and disability were significantly lower; however, many postoperative complications which are the main problems of neurosurgeon should still be controlled. There are many complications caused by the residual cavity of giant tumor which include acute subdural hematoma (SDH), remote epidural hematoma (EDH), residual cavity hematoma $(\mathrm{RCH})$, ischemic cerebral infarction, subcu- taneous and tumor cavity tension effusion. The Foley balloon catheter technique was applied in 181 patients and good outcome was achieved after the resection of giant intracranial tumor.

\section{Postoperative complications}

The complications after surgery of giant intracranial tumor are caused by its own particularity, much blood and cerebrospinal fluid loses during the longer time surgery relative to the resection of regular tumor (Left in Figure 2). Intracranial tissues cannot rise quickly after they have been compressed for a long time though space-occupying is relieved. SDH is subjected to stretching of the subdural bridging veins and venous drainage which caused by cortex collapse [6]. Hyo SJ, et al. [7] reported a case of spinal subdural hematoma following the operation of meningioma removal. The pathogenesis of the complication includes the downward movement of blood from the cranial compartment by gravity, excessive decompression of intracranial space during surgery and the lowered cerebrospinal fluid (CSF) pressure by drainage. In addition, remote EDH happened due to intracranial shift by gravity and lower tension. Most authors [8] have agreed that the loss of CSF is the underlying reason for EDH which are of meningeal artery and vein, this patho-mechanism can be as follows:

1) Mechanical causes such as the displacement of cerebellum after CSF loss stretch and tear the cerebral veins. Besides, RCH appears when the blood flows to the residual cavity. Most common mechanisms involve rupture of the abnormal vascular networks around the tumor, such as weak thin walled vessels and direct peritumoral vascular erosion by the tumor [9].

2) Capillary was susceptible to rupture under stress because enlarged tortuous feeding arteries are less resistant to cerebral perfusion pressure breakthrough.

3) Based on the hemodynamic theory by Koing, et al. [10] the removal of a supratentorial space occupying mass may induce a reduction in intracranial pressure with a critical increase in the transmural pressure of vein or venule. Another mechanism of this association is vasoactive substances released by the tumor [11]. Ischemic cerebral infarction sometimes occurs because of the tortuosity occlusion of important vessels by cortex collapse. Besides, other complications come up including intracranial pneumatocele, subcutaneous or tumor cavity tension effusion.

\section{Creativity}

The insertion of Foley balloon catheter with balloon inflation in the tumor cavity is one of the time-tested methods 

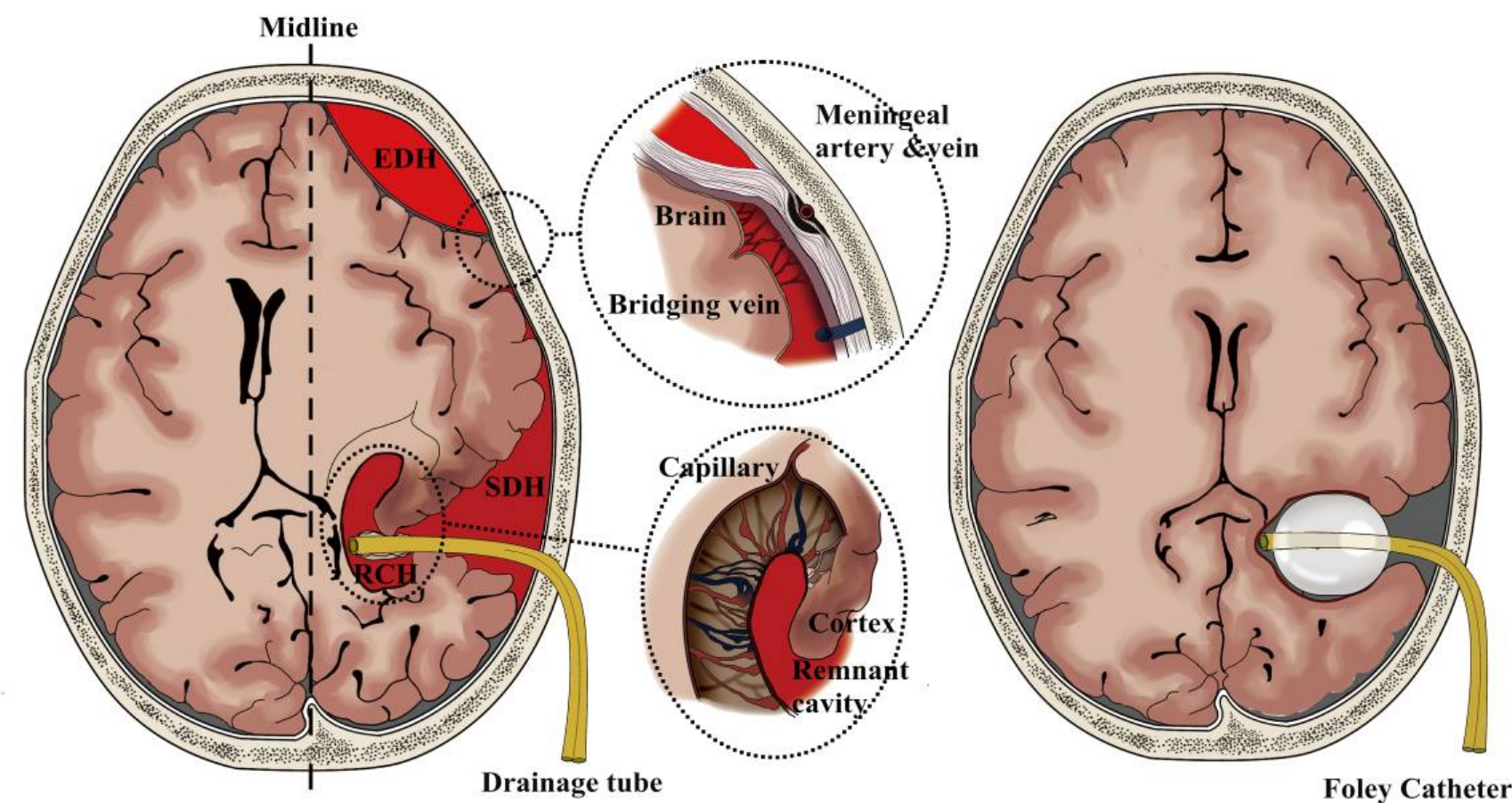

Foley Catheter

EDH: Epidural hematoma; SDH: Subdural hematoma; RCH: Remnant Cavity hematoma.

Figure 2: The Schematic drawing of the difference with and without a Foley balloon catheter.

Left: Postoperative complications of EDH, SDH and RCH without the migration of Foley balloon catheter. EDH happened because of meningeal artery and vein ruptured due to the loss of CSF, gravity and low tension of brain; SDH is subjected to stretching of the bridging and drainage veins that cause by cortex collapse (Middle-upper). RCH is original of the blood flow from the SDH and cerebral pressure breakthrough (Middle-lower); Right: No complications with Foley balloon catheter, the capsule gives support to the tumor cavity around.
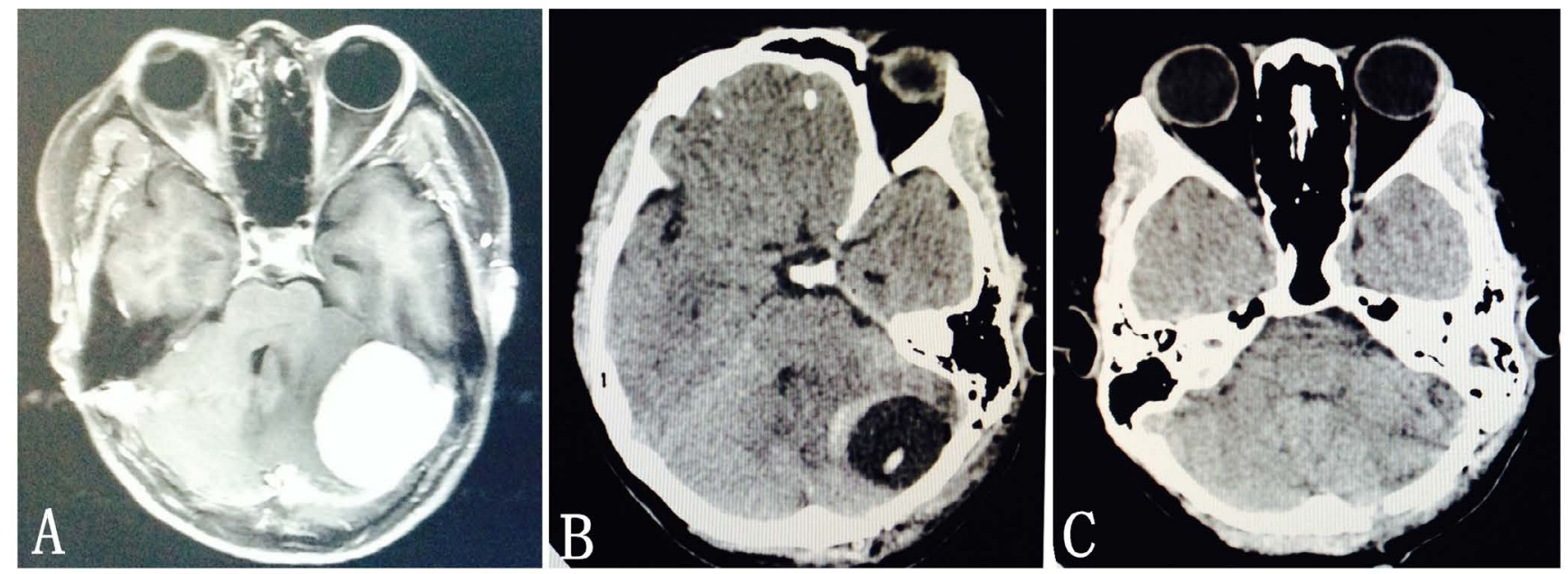

Figure 3: Foley catheter applied in operation of subratentorial tumor.

A) MRI of axial contrast head demonstrating a giant tumor in the posterior cranial fossa; (B,C) CT scan after operation, B) Foley balloon catheter was clearly seen on CT scan 1 day postoperatively; C) No complications were observed on CT scan 3 days after surgery.

despite the fact that this catheter are neither recommended nor designed for this purpose. In the medical literature [12] there described 4 cases of intracranial migration of Foley balloon catheter which was related to profuse epistaxis after severe craniofacial trauma and restricted to anterior and middle tank. Giannantonio reported inflated Foley balloon catheter in the cranium as a self-retaining retractor which can protect the cerebral cortex with more elasticity, less trauma and easy adjustability [13]. Shahid M Nimjee presented a case with tension pneumocephalus who were treated by inserting a Foley balloon catheter [14]. 
Citation: Yang L, Wu T, Cui J, et al. (2019) The Effect of Intracranial Migration of Foley Balloon Catheter after Tumor Resection. J Neurosurg Res $\operatorname{Rev} 2(1): 27-32$

The advantages of intracranial migration of Foley balloon catheter are as follows (Right in Figure 2):

1) It can reset the collapse of intracranial cortical tissue, relieve distortion draining veins, and pull the bridge vein and the supplying arteries. It can also prevent the formation of ischemic infarction and/or acute cerebral edema by restoring blood supply and drainage of the effusion.

2) Intracranial pressure (ICP) is controlled falling down slowly by extracted saline from the Foley balloon catheter so as to prevent EDH due to remote epidural stripping.

3) It can resist the cerebral perfusion pressure breakthrough around the cavity and reduce the capillary bleeding by expanded Foley balloon catheter.

4) Foley balloon catheter can serve as a drainage system which is independent of the balloon.

\section{Indications and surgical considerations}

No postoperative complication and infection is found with this technique. The Foley balloon catheter was pulled out after gradual steady traction and repeat $\mathrm{CT}$ scan showed no other findings (Figure 3, Figure 4 and Figure 5). The indications of this technique are as follows:

1) Intracranial veins around tumor resection, such as bridge vein, intracranial vein, Labbe vein, cortical vein, et al. should be protected as far as possible. Our experience is freeing up sufficient space to fully loosen the veins so as to prevent the occurrence of postoperative intractable intracranial edema.

2) The balloon surface is smooth and slippery, which facilitates intracranial insertion. However, no sticking or adherence to cortex or interior wall of tumor should be ensured.
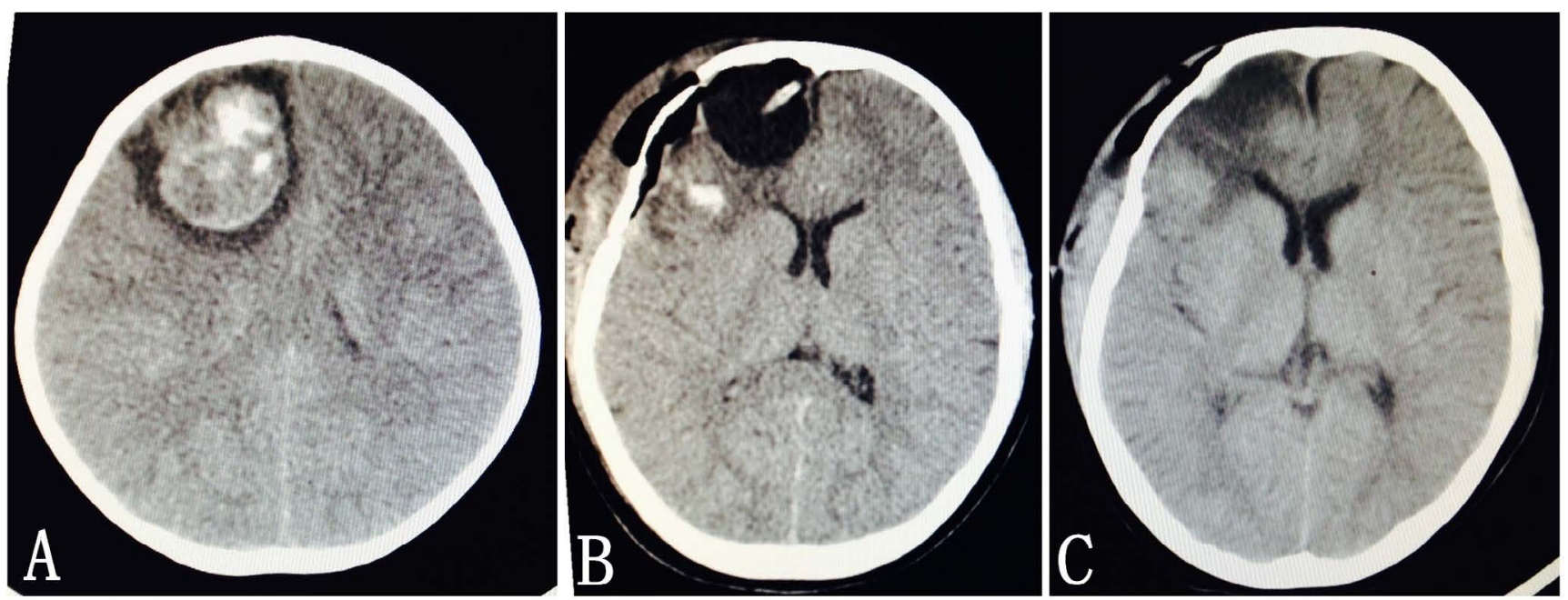

Figure 4: Foley catheter applied in operation of supratentorial tumor.

A) Axial non-contrast head CT demonstrating a giant tumor in right frontal lobe; B,C) CT scan after operation, B) Foley balloon catheter was clearly seen on CT scan 24 hours after surgery; C) No complications were observed on CT scan 2 days post-operation.
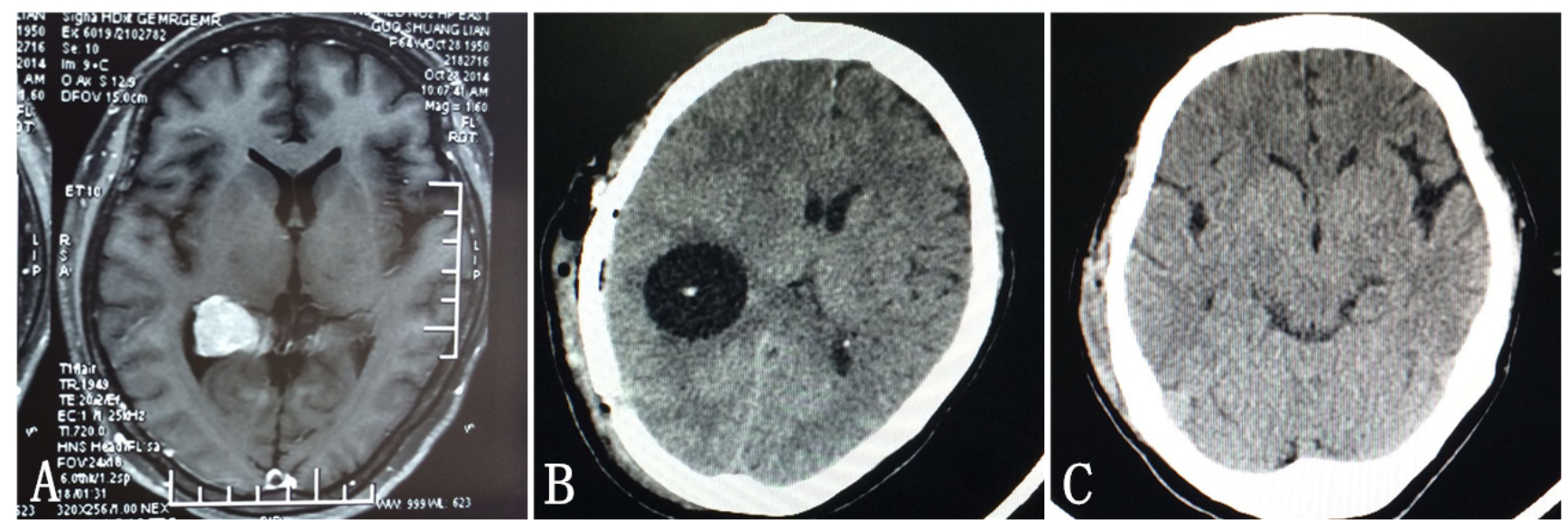

Figure 5: Foley catheter applied in operation of supratentorial tumor.

A) Axial contrast head MRI demonstrating a giant tumor (ependymocytoma by pathology) close to right ventricle; B,C) CT scan after operation, B) Foley balloon catheter was clearly seen on CT scan 1 day after surgery; C) No complications were observed on CT scan 2 days post-operation. 
That is why Surgicel ${ }^{\circledR}$ is needed before the arrangement of Foley balloon catheter. In addition, the mechanism of hematoma formation associated with the Valsalva maneuver is poorly understood but Shojima, et al. mentioned that the events such as seizure or a rapid change in blood pressure might also act as an etiologic agent for hemorrhage [11]. Amit Agrawal, et al. [15] reported a case of diffuse subarachnoid hemorrhage following posterior fossa meningioma surgery. He stressed the importance of early postoperative CT scan and the optimal management of ventricular pressure and also detected that the prevention of coagulation abnormalities will possibly prevent this life-threatening complication. It has been suggested that CT scan should be performed for any patient who has declining level of consciousness after surgery so as to establish the diagnosis. Furthermore, it is recommended to avoid rapid CSF loss both during and after surgery. Blood pressure need to be monitored continuously, early detection and awareness of this complication may help to avoid unnecessary interventions.

\section{Conclusions}

In summary, all the patients in our study were recovered in different degrees without any residual cavity complication. Intracranial migration of Foley balloon catheter can effectively reduce the risk of postoperative complications such as intracranial hemorrhage, acute intracranial edema and ischemic cerebral infarction. The success of this technique with a simple method in a series of our cases has encouraged its further use.

\section{Acknowledgements}

This project was supported by foundation of the Second Hospital of Hebei Medical University (Grant No. ZH1201302) (Zhenzeng Fan).

\section{Disclosure}

The authors report no conflict of interest concerning the materials or methods used in this study or the findings specified in this paper.

\section{Author Contributions}

Author contributions to the study and manuscript preparation include the following:

Conception and design: ZF and LY.

Acquisition of data: FW, JC and TW.

Analysis and interpretation of data: all authors.

Drafting the article: LY and JW.

Critically revising the article: all authors.

Reviewed submitted version of manuscript: all authors.
Approved the final version of the manuscript on behalf of all authors.

Statistical analysis: LY, GZ.

Study supervision: ZF and LY.

\section{References}

1. Rutka JT, Kim B, Etame A, et al. (2014) Nanosurgical resection of malignant brain tumors: Beyond the cutting edge. ACS Nano 28: 9716-9722.

2. Jung MH, Moon KS, Lee KH, et al. (2014) Surgical experience of infratentorial meningiomas: Clinical series at a single institution during the 20-year period. J Korean Neurosurg Soc 55: 321-330.

3. Turkoglu E, Gurer B, Sanli AM, et al. (2013) Clinical outcome of surgically treated low-grade gliomas: A retrospective analysis of a single institute. Clin Neurol Neurosurg 115: 2508-2513.

4. Li TY, Chu JS, Xu YL, et al. (2014) Surgical strategies and outcomes of spinal ependymomas of different lengths: Analysis of 210 patients: Clinical article. J Neurosurg Spine 21: 249-259.

5. Noorbakhsh A, Tang JA, Marcus LP, et al. (2014) Gross-total resection outcomes in an elderly population with glioblastoma: A seer-based analysis. J Neurosurg 120: 31-39.

6. Kashimura H, Arai H, Ogasawara K, et al. (2008) Lipomatous meningioma with concomitant acute subdural hematoma--case report. Neurol Med Chir (Tokyo) 48: 466-469.

7. Jun HS, Oh JK, Park YS, et al. (2014) Spinal subdural hematoma following meningioma removal operation. Korean J Spine 11: 12-14.

8. Baeesa SS (2012) Remote cerebellar hemorrhage in neurosurgery. Neurosciences (Riyadh) 17: 305-308.

9. Bloomgarden GM, Byrne TN, Spencer DD, et al. (1987) Meningioma associated with aneurysm and subarachnoid hemorrhage: Case report and review of the literature. Neurosurgery 20: 24-26.

10. König A, Laas R, Herrmann HD (1987) Cerebellar haemorrhage as a complication after supratentorial craniotomy. Acta Neurochir (Wien) 88: 104-108.

11. Worm PV, Ferreira MP, Ferreira NP, et al. (2009) Subdural haematoma in a patient with meningioma. Arq Neuropsiquiatr 67: 308-310.

12. Veeravagu A, Joseph R, Jiang B, et al. (2013) Traumatic epistaxis: Skull base defects, intracranial complications and neurosurgical considerations. Int J Surg Case Rep 4: 656-661.

13. Spena G, Versari P (2011) Bubbles in the head: A new method for brain retraction during craniotomy. Acta Neurochir (Wien) 153: 1807-1811.

14. Nimjee SM, Zomorodi AR, Adamson DC (2011) Conquering mount fuji: Resolution of tension pneumocephalus with a foley urinary catheter. Case Rep Radiol 2011: 164316.

15. Agrawal A, Kakani A, Ray K (2010) Extensive supratentorial hemorrhages following posterior fossa meningioma surgery. $J$ Surg Tech Case Rep 2: 87-89.

DOI: $10.36959 / 363 / 409$

Copyright: (C) 2019 Yang L, et al. This is an open-access article distributed under the terms of the Creative Commons Attribution License, which permits unrestricted use, distribution, and reproduction in any medium, provided the original author and source are credited. 